\title{
Anti-allergic effect of Lactobacillus helveticus SBT2171 on pollen allergen-induced allergy model
}

\author{
Maya Yamashita*, Kurumi Matsumoto, Nanae Matsumoto, Eiji Kobatake, Toshihide \\ Kabuki
}

Milk Science Research Institute, Megmilk Snow Brand Co. Ltd., 1-1-2, Minamidai, Kawagoe, Saitama, 350-1165, Japan

Corresponding author: Maya Yamashita, $\mathrm{PhD}$, Milk Science Research Institute, Megmilk Snow Brand Co. Ltd., 1-1-2, Minamidai, Kawagoe, Saitama, 350-1165, Japan.

Submission date: January $18^{\text {th }}$, 2019, Acceptance Date: March $28^{\text {th }}$, 2019, Publication Date: March $31^{\text {st }}, 2019$

Citation: Yamashita M., Matsumoto K., Matsumoto N., Kobatake E., Kabuki T. Effects of daily intake of Harudori-kombu: Anti-allergic effect of Lactobacillus helveticus SBT2171 on pollen allergen-induced allergy model. Functional Foods in Health and Disease 2019; 9(3): 166-179. DOI: https://doi.org/10.31989/ffhd.v9i3.586

\begin{abstract}
Background: We recently reported the suppressive effects of Lactobacillus helveticus SBT2171 (LH2171) on the proliferation and inflammatory cytokine production of primary murine immune cells in vitro. In this study, the aim was to investigate the effect of LH2171 on a murine pollen allergen- induced allergy model.
\end{abstract}

Methods: Male BALB/c mice were fed a diet containing LH2171 (LH2171 group) or a diet not containing LH2171 (control group). Then, pollen allergy was induced by immunization with pollen extract emulsified in alum adjuvant and intranasal administration of pollen extract. To evaluate the anti-allergenic effect of LH2171, the antibody titer in plasma and expression of cytokines and transcription factors in immune tissues were analyzed by ELISA and qRT-PCR.

Results: In the LH2171 group, the amounts of pollen-specific antibody and total IgE in plasma were significantly decreased compared with those in the control group. Proliferation and Th2 cytokine production were lower in the submandibular lymph node cells of the LH2171 group than 
in those of the control group. Moreover, in Peyer's patches, the gene expression of 1110 and Foxp3 were increased in the LH2171 group.

Conclusion: LH2171 has the ability to suppress production of allergy-related antibody and Th2 cytokines, which could be correlated with the induction of anti-inflammatory factors in the intestinal immune system.

\section{BACKGROUND}

In recent years, allergies to pollen and household dust have rapidly increased worldwide, including in Japan, and are becoming serious social concerns [1-3]. These allergies are commonly treated with oral antihistamines and steroids, but long-term use of these medications can carry risks of serious side effects such as drowsiness, gastrointestinal disorders, dry mouth, dizziness, headache, or infections. Furthermore, the effectiveness of these drugs is limited to a short window, only the period immediately after the onset of allergy. Therefore, foods that effectively prevent allergic reactions that can be safely ingested on a daily basis for long periods of time are strongly desired.

Lactic acid bacteria (LAB) is a general term used to describe bacteria that produce lactic acid as a metabolite. LAB have long been used for the fermentation of foods such as yogurt, fermented milk, cheese, and pickles and for improving the shelf life, nutritional value, and palatability of foods, allowing for the enrichment of consumer diets. Some strains of LAB naturally exist in the human gastrointestinal tract - including the intestines - as well as in the vagina and are known to contribute to homeostatic maintenance of the gut environment if compromised by pathogenic microorganisms [4, 5]. Moreover, not only live LAB, but dead LAB are also known to act directly and indirectly on the physiological health of the host, through modulation of intestinal microbiota as a biogenic [6].

Recently, LAB have been reported to not only improve the intestinal environment, but also to influence immune function. For instance, as immunostimulators, LAB confer protection against viral infection [7, 8] and induce IgA production, enhancing host immune defense [9]. In addition, LAB are capable of modulating excessive immune responses through anti-allergic action $[10,11]$ and autoimmune disease-mitigating action [12-14].

Lactobacillus helveticus SBT2171 (LH2171), used in this study, is a LAB isolated from dairy products and has high protease activity, therefore, it is commonly used as a starter bacterial strain in the production of Gouda-type cheeses [15]. Our previous study clarified that LH2171 significantly suppressed the proliferation of primary murine immune cells among 41 LAB strains of various species and decreased the production of lipopolysaccharide (LPS)-stimulated inflammatory cytokines from the immune cells in vitro [16]. In addition, LH2171 prevented the development of rheumatoid arthritis [17, 18] and multiple sclerosis [19]-major autoimmune diseases - in a murine model in vivo. These reports suggested that LH2171 has beneficial immunoregulatory properties.

In the present study, to further investigate the beneficial properties of LH2171 in modulating excessive immune reactions, we evaluated its alleviating effects in a pollen allergen-induced 
allergy model. This could contribute to elucidating the mechanisms by which LAB regulates immune function and contribute to the promotion and development of LAB-based prevention or treatment strategies for allergy diseases.

\section{METHODS}

\section{Preparation of LH2171 cells}

L. helveticus SBT2171 (LH2171) was isolated by Megmilk Snow Brand Co., Ltd. (Tokyo, Japan), inoculated into Lactobacilli MRS broth (BD Biosciences, San Jose, CA, USA), and cultivated for $16 \mathrm{~h}$ at $37^{\circ} \mathrm{C}$. Following incubation, the cells were harvested by centrifugation at $8,000 \times \mathrm{g}$ for 10 $\min$ at $4{ }^{\circ} \mathrm{C}$. The cells were washed twice with $0.9 \%$ saline and once with distilled water and freezedried $\left(3.1 \times 10^{10} \mathrm{cfu} / \mathrm{g}\right.$ in powder form) .

\section{Administration of LH2171 and generation of pollen allergen-induced allergy mouse model}

Male BALB/c mice, aged 6 weeks, were purchased from CLEA Japan, Inc. (Tokyo). The mice received deionized water and diet ad libitum and were maintained under controlled conditions of temperature, humidity, and light $\left(23 \pm 2^{\circ} \mathrm{C}, 50 \pm 10 \%\right.$ humidity, and 12-h light-dark cycle $)$. All experiments involving mice were carried out in accordance with the animal experimentation regulations of the Milk Science Research Institute of Megmilk Snow Brand Co., Ltd., which are based on the guidelines proposed by the Science Council of Japan and the National Institutes of Health Guide for the Care and Use of Laboratory Animals, and were approved by the Animal Care and Use Committee of Milk Science Research Institute, Megmilk Snow Brand Co., Ltd.

The mice were acclimatized to the American Institute of Nutrition (AIN)-93G diet [20] for 1 week and then divided into three groups: mice fed AIN-93G without the induction of pollen allergy (normal group), mice fed AIN-93G with the induction of pollen allergy (control group), or mice fed AIN-93G containing LH2171 with the induction of pollen allergy (LH2171 group). Compositions of the experimental diets are shown in Table 1. After experimental diet intake for 1 week, the control and LH2171 groups were immunized by intraperitoneal injection of $100 \mu \mathrm{g}$ cedar pollen extract (Cosmo Bio, Tokyo, Japan) and 0.1 $\mu \mathrm{g}$ recombinant mouse IL-4 (R\&D Systems, Minneapolis, MN, USA), which were dissolved in $75 \mu \mathrm{L}$ of phosphate-buffered saline (PBS) and emulsified in $125 \mu \mathrm{L}$ of alum adjuvant (Thermo Fisher Scientific, Waltham, MA, USA), to induce pollen allergy. Fourteen and twenty-eight days after the first immunization, mice were boosted by intraperitoneal injection of $100 \mu \mathrm{g}$ cedar pollen extract, which was dissolved in $75 \mu \mathrm{L}$ of PBS and emulsified in $125 \mu \mathrm{L}$ of alum adjuvant and administered $10 \mu \mathrm{L}$ pollen solution $(2 \mathrm{mg} / \mathrm{mL})$ daily via nostrils from 35 days to 41 days after the first immunization. The experimental schedules are shown in Figure 1. The antigen-specific antibody titer and total IgE level in plasma, cell proliferation, and cytokine production of submandibular lymph node and spleen cells were evaluated at 42 days, and the expression of allergy-related cytokines and transcription factors in the Peyer's patch was evaluated at 21 days. 
Table 1. Compositions of the experimental diets.

\begin{tabular}{lrr}
\hline & \multicolumn{2}{c}{$(\%, \mathrm{w} / \mathrm{w})$} \\
Ingredient & AIN-93G & AIN-93G+LH2171 \\
\hline \hline Casein & 20 & 20 \\
L-cystine & 0.3 & 0.3 \\
Cornstarch & 40 & 39 \\
a-Cornstarch & 13.2 & 13.2 \\
Sucrose & 10 & 10 \\
Soybean oil & 7 & 7 \\
Cellulose & 5 & 5 \\
Mineral mix (AIN-93G-MX) & 3.5 & 3.5 \\
Vitamin mix (AIN-93-VX)* & 1 & 1 \\
t-Butylhydroquinone & 0.0014 & 0.0014 \\
Lactobacillus helveticus SBT2171 & - & 1 \\
\hline Total & 100 & 100 \\
\hline
\end{tabular}

*AIN-93G vitamin mixture containing choline bitartrate

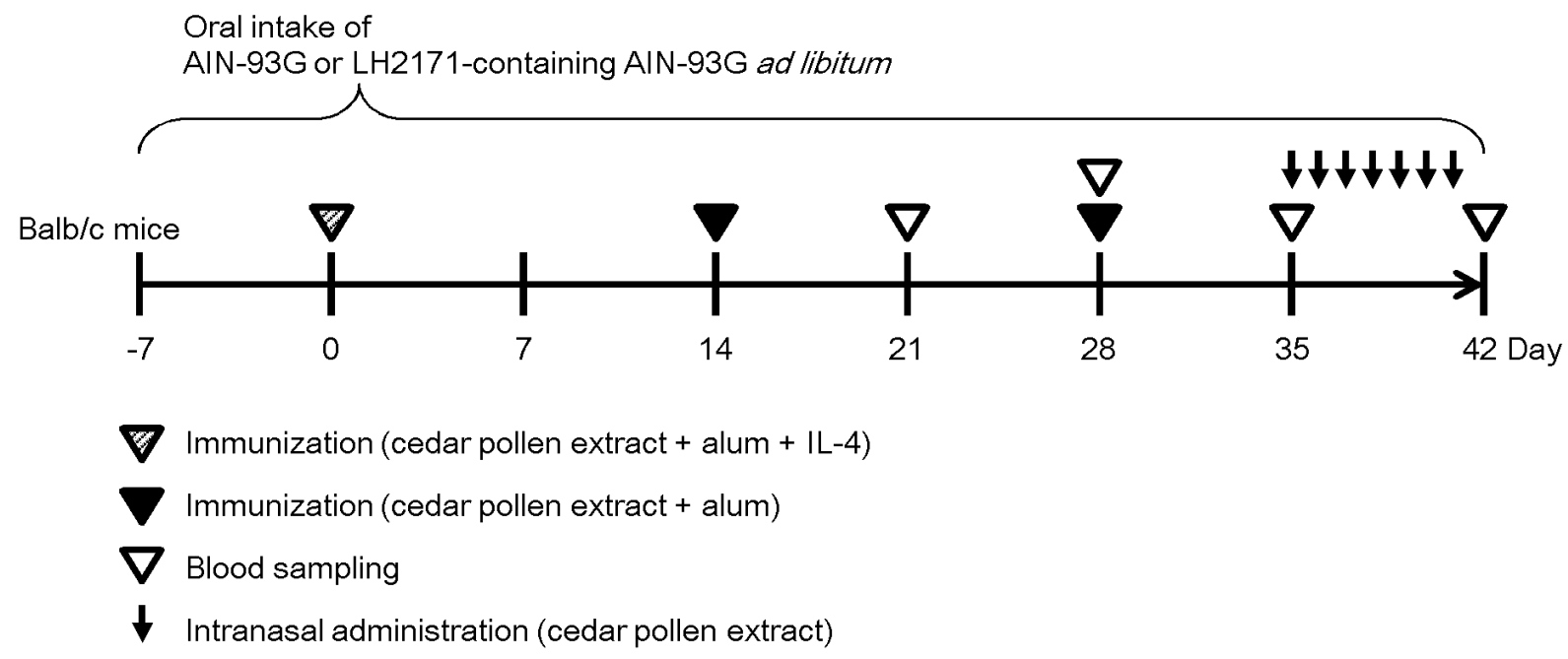

Figure 1. Experimental design of pollen-induced allergy in a mouse model. Male BALB/c mice (7 weeks old) were divided into the normal (not pollen sensitized), control (pollen sensitized), or LH2171 group (LH2171 administered and pollen sensitized). To induce an allergic response, the control and LH2171 groups were immunized by intraperitoneal injection of $100 \mu \mathrm{g}$ cedar pollen extract and $0.1 \mu \mathrm{g}$ recombinant mouse IL-4, adsorbed in alum. Fourteen and twenty-eight days after the first immunization, the mice were boosted by intraperitoneal injection of $100 \mu \mathrm{g}$ cedar pollen extract adsorbed in alum. Sensitization was followed by daily intranasal administration of pollen extract for 7 days, 1 week after the third immunization. The LH2171-containing diet was fed to the LH2171 group ad libitum from the beginning to the end of the experiment. 


\section{Measurement of antigen-specific antibody titer and total IgE level in plasma}

The antigen-specific antibody titer was determined by the modified enzyme-linked immunosorbent assay (ELISA) as previously described [21]. In brief, immunoplates (Corning, NY, USA) were coated with a solution containing $100 \mu \mathrm{g} / \mathrm{mL}$ of cedar pollen extract and incubated at $4^{\circ} \mathrm{C}$ overnight. After blocking with $1 \%$ bovine serum albumin (Merck Millipore, Burlington, MA, USA) in PBS, plasma samples were added into the pollen-coated wells, and the plates were incubated for $1 \mathrm{~h}$ at room temperature (RT). After five washes with $0.05 \%$ Tween 20 (Wako Pure Chemical Industries, Osaka, Japan) in PBS (Tween-PBS), the plates were incubated with horseradish peroxidase-conjugated anti-mouse IgG1 antibody (SouthernBiotech, Birmingham, AL, USA) for $1 \mathrm{~h}$ at RT. Following five washes with Tween-PBS, the plates were developed with QuantaBlu $^{\text {TM }}$ Fluorogenic Peroxidase Substrate Kit (Thermo Fisher Scientific). According to the manufacturer's protocol, the fluorescence intensity of each well was measured by Varioskan ${ }^{\circledR}$ Flash (Thermo Fisher Scientific). The total IgE level in the plasma was evaluated using the Mouse IgE ELISA Quantitation Set (Bethyl Laboratories, Montgomery, TX, USA) according to the manufacturer's protocol.

\section{Cell proliferation assay and cytokine analysis}

At day 42 after the first immunization, the spleen (SP) and submandibular lymph nodes (LNs) of the mice were collected and mechanically disrupted in RPMI 1640 culture medium (GIBCO; Thermo Fisher Scientific) containing 10\% heat-inactivated fetal bovine serum (GIBCO), $10 \mathrm{mM}$ HEPES buffer (GIBCO), $100 \mathrm{U} / \mathrm{mL}$ penicillin (GIBCO), $100 \mu \mathrm{g} / \mathrm{mL}$ streptomycin (GIBCO), and $0.05 \mathrm{mM}$ 2-mercaptoethanol. Cell suspensions were filtered, and erythrocytes remaining in the SP cell preparation were eliminated by lysis buffer $\left(1.68 \mathrm{M} \mathrm{NH}_{4} \mathrm{Cl}, 99.9 \mathrm{mM} \mathrm{KHCO}_{3}\right.$, and $0.818 \mathrm{mM}$ EDTA). Cells were washed twice, resuspended in the cell culture medium, and stored on ice until the cell culture experiments.

To assay cell proliferation, the cells isolated from mice were stimulated with cedar pollen extract in 96-well plates $\left(2 \times 10^{5}\right.$ cells/well; IWAKI; Asahi Techno Glass, Chiba, Japan $)$ at $37^{\circ} \mathrm{C}$ for 5 days with $5 \% \mathrm{CO}_{2}$. After stimulation, PrestoBlue Cell Viability Reagent (Life Technologies, Frederick, MD, USA), which exhibits cellular proliferation-dependent fluorescence, was added to each well according to the manufacturer's protocol and incubated at $37^{\circ} \mathrm{C}$ for about $3 \mathrm{~h}$ with $5 \%$ $\mathrm{CO}_{2}$. After incubation, the fluorescence intensity (excitation: $530 \mathrm{~nm}$; emission: $580 \mathrm{~nm}$ ) of each well was measured.

To measure the production of allergy-related cytokines from immune cells, the cells isolated 
from mice were stimulated with $100 \mu \mathrm{g} / \mathrm{mL}$ cedar pollen extract in 24 -well plates $\left(1.5 \times 10^{6}\right.$ cells/well; IWAKI) at $37^{\circ} \mathrm{C}$ for 5 days with $5 \% \mathrm{CO}_{2}$. After stimulation, the media of cultured immune cells were harvested and stored at $-80^{\circ} \mathrm{C}$. The secretion of IL-4, IL-5, IL-10, IL-13, and IFN- $\gamma$ in the culture cell media was measured with ELISA kits (IL-5, IL-10, and IL-13: eBioscience, San Diego, CA, USA; IL-4 and IFN- $\gamma$ : BioLegend, San Diego, CA, USA). The cytokine concentrations were evaluated according to the manufacturers' protocols.

\section{Gene expression analysis}

Total RNA was isolated from the Peyer's patches (PP) of mice using the RNeasy Mini Kit (Qiagen, Valencia, CA, USA). RNA was reverse-transcribed into complementary DNA using the ReverTra Ace qPCR RT Master Mix with gDNA Remover (TOYOBO, Osaka, Japan). Real-time polymerase chain reaction (PCR) was performed using the TaqMan ${ }^{\circledR}$ Fast Advanced Master Mix (Life Technologies) and the fluorescent TaqMan ${ }^{\circledR}$ probe specific for murine IFN- $\gamma$ (Mm01168134-m1), mIL-4 (Mm00445259-m1), mIL-5 (Mm00439646-m1), mIL-13 (Mm00434204-m1), mIL-10 (Mm00439614-m1), mFoxp3 (Mm00475165-m1), or the housekeeping gene mGAPDH (Mm99999915-g1) (Life Technologies) according to the manufacturer's protocols. Data were normalized to Gapdh gene expression.

\section{Statistical analysis}

Student's $t$-test was used to compare the control and LH2171 groups. All analyses were performed using StatView version 5.0 (SAS Institute, Cary, NC, USA). $P$ values $<0.05$ were considered statistically significant.

\section{RESULTS}

\section{Effect of LH2171 on antigen-specific antibody titer and total IgE level in plasma}

At day 42 after the first immunization, the amount of antigen-specific IgG1 in plasma was significantly lower in LH2171 mice than in the control mice (Fig. 2A). Moreover, total IgE was also lower in the plasma obtained from the LH2171 group than in plasma from the control group at day 21 and 35 (Fig. 2B). In the plasma of the normal group, which was not immunized with cedar pollen, antigen-specific IgG1 and total IgE were not detected. 
(A)

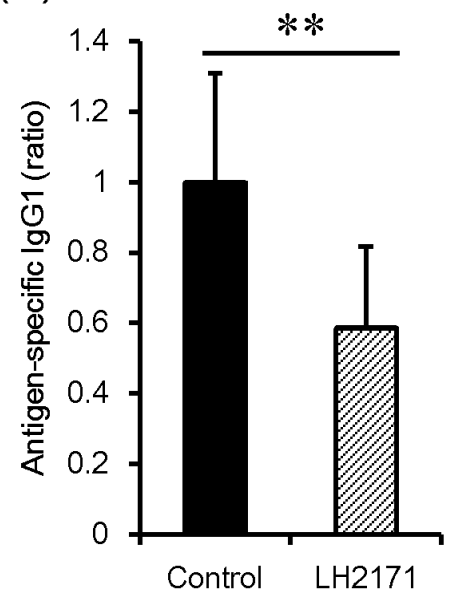

(B)

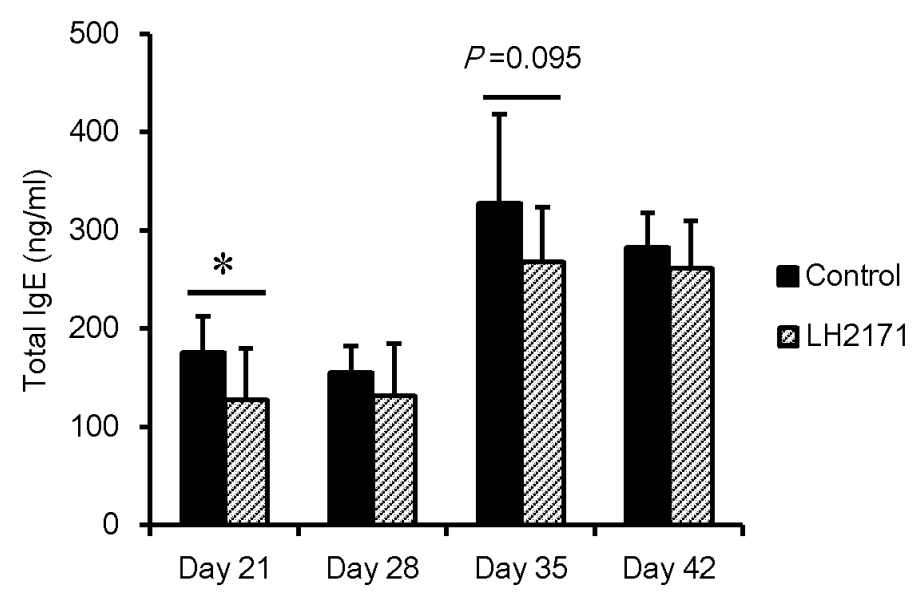

Figure 2. Effect of LH2171 on antigen-specific antibody titer and total IgE level in plasma of pollen-sensitized mice. (A) Antigen-specific IgG1 levels and (B) total IgE levels in plasma of the mice 42 days after the first immunization. Antigen-specific IgG1 and total IgE were measured by ELISA. Data are shown as the means $\pm \mathrm{SD}$ (normal group, $\mathrm{n}=3$; control group, $\mathrm{n}=10$; LH2171 group, $\mathrm{n}=10$ ). The LH2171 group was compared with the control group by Student's $t$-test $\left({ }^{*} P<0.05,{ }^{* *} P<0.01\right)$.

\section{Effect of LH2171 on the proliferation of submandibular LN and SP cells}

The proliferative responses of the immune cells isolated from the submandibular LNs, which are responsible for draining LNs of the nose, through which the antigen was administered in this study, were significantly suppressed in mice administered LH2171 compared with those of the control mice at day 42 after the first immunization (Fig. 3A). In contrast, there was no significant difference in the proliferation of immune cells from the SP, which controls the whole-body immune system, between LH2171-administered and control mice (Fig. 3B).

(A)

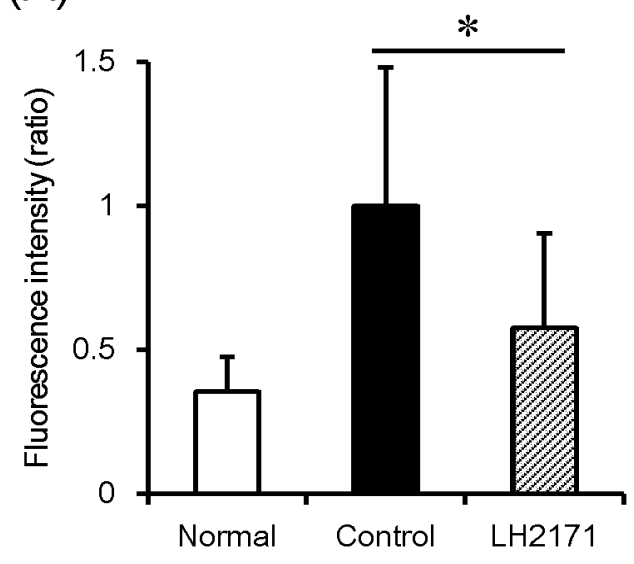

(B)

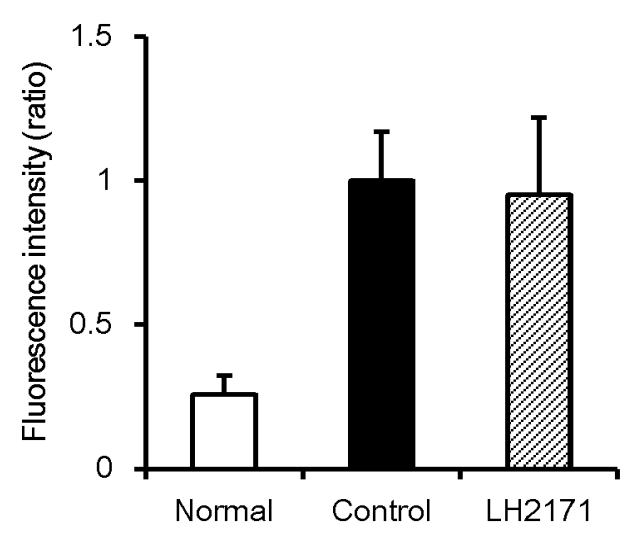

Figure 3. Effect of LH2171 on proliferation of immune cells from pollen-sensitized mice stimulated with pollen extract. Immune cells from the submandibular lymph nodes (A) and spleen (B) 
of mice 42 days after the first immunization were cultured with pollen extract $(100 \mu \mathrm{g} / \mathrm{mL})$ for 5 days. After cultivation, the cell proliferation rate was measured using the PrestoBlue Cell Viability Reagent and represented as fluorescence intensity (\%), in which the value obtained from the control group was set to $100 \%$. Data are shown as the means $\pm \mathrm{SD}$ (normal group, $\mathrm{n}=3$; control group, $\mathrm{n}=10$; LH2171 group, $\mathrm{n}=$ 10). The LH2171 group was compared with the control group by Student's $t$-test $\left({ }^{*} P<0.05\right)$.

\section{Effect of LH2171 on allergy-related cytokine production by submandibular LN cells}

The analysis of cytokine levels (IL-4, IL-5, IL-13, IFN- $\gamma$, and IL-10) in the culture media of the submandibular LN cells by ELISA showed that the IL-5 level was significantly lower (Fig. 4), and the IL-13 level had a tendency to be lower in mice administered LH2171 ( $P=0.083$; Fig. 4) compared with those of the control mice at day 42 after the first immunization. On the other hand, there was no significant difference in the IFN- $\gamma$ level between the LH2171 and control mice. The IL-10 level tended to decrease in LH2171 mice compared with that in the control mice $(P=0.061$; Fig. 4).

\section{Effect of LH2171 on the expression of allergy-related cytokines and transcription factors in PP} Analysis of cytokines and transcription factors related to allergy symptoms in the PP at 21 days after the first immunization showed a significant increase in IL-10 and Foxp3 expression levels in LH2171 mice (Fig. 5). On the other hand, there was no statistically significant difference in the expression of IL-4, IL-5, IL-13, and IFN- $\gamma$ between LH2171 mice and control mice.
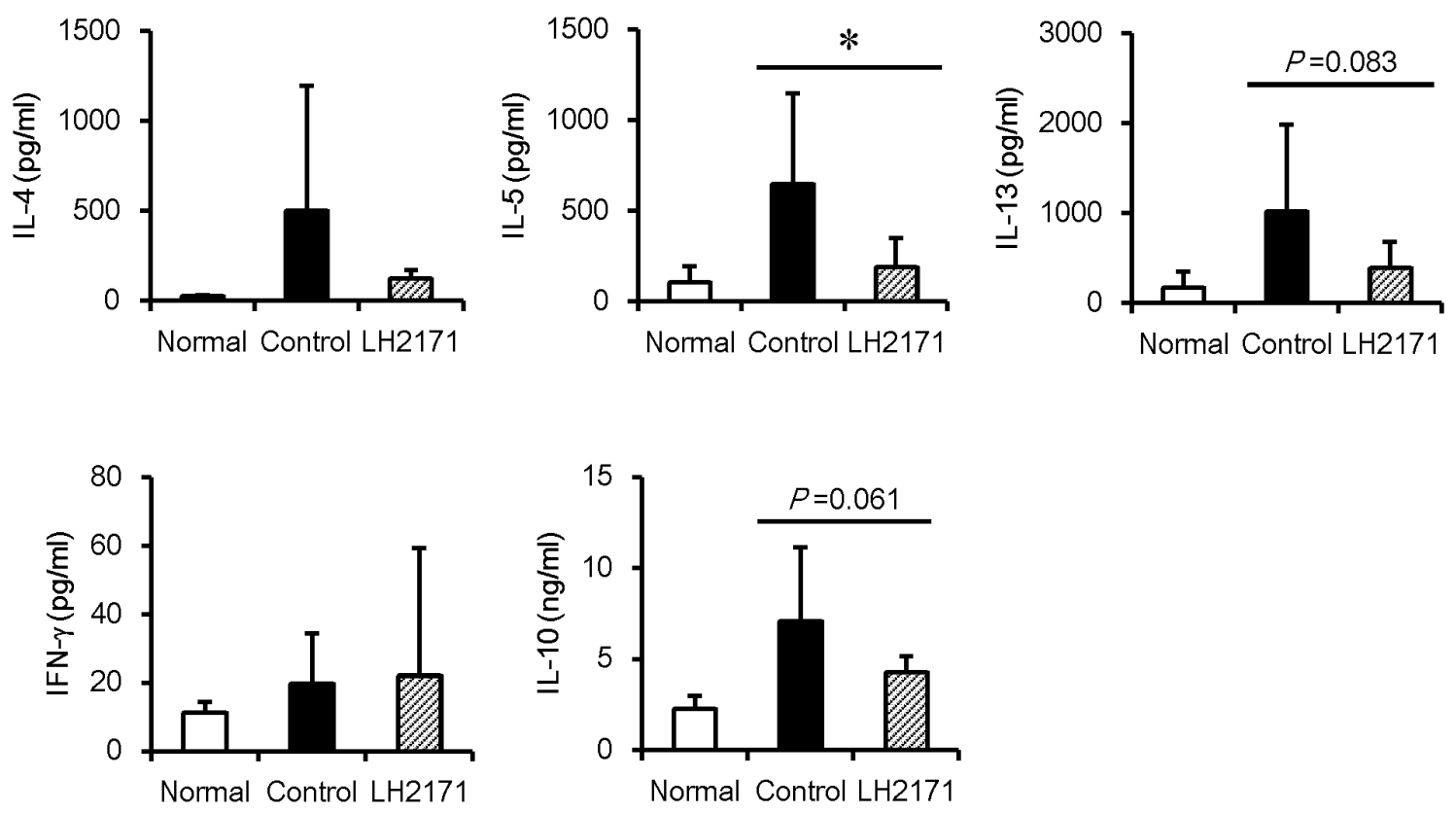

Figure 4. Effect of LH2171 on allergy-related cytokines produced by pollen-stimulated lymph node cells from pollen-sensitized mice. Immune cells from submandibular lymph nodes of mice 42 days after the first immunization were cultured with pollen extract $(100 \mu \mathrm{g} / \mathrm{mL})$ for 5 days. After cultivation, 
their media were harvested, and the concentration of IL-4, IL-5, IL-10, IL-13, and IFN- $\gamma$ in the media was measured with ELISA. Data are shown as the means $\pm \mathrm{SD}$ (normal group, $\mathrm{n}=3$; control group, $\mathrm{n}=10$; LH2171 group, $\mathrm{n}=10)$. The LH2171 group was compared with the control group by Student's $t$-test $(* P<$ $0.05)$.

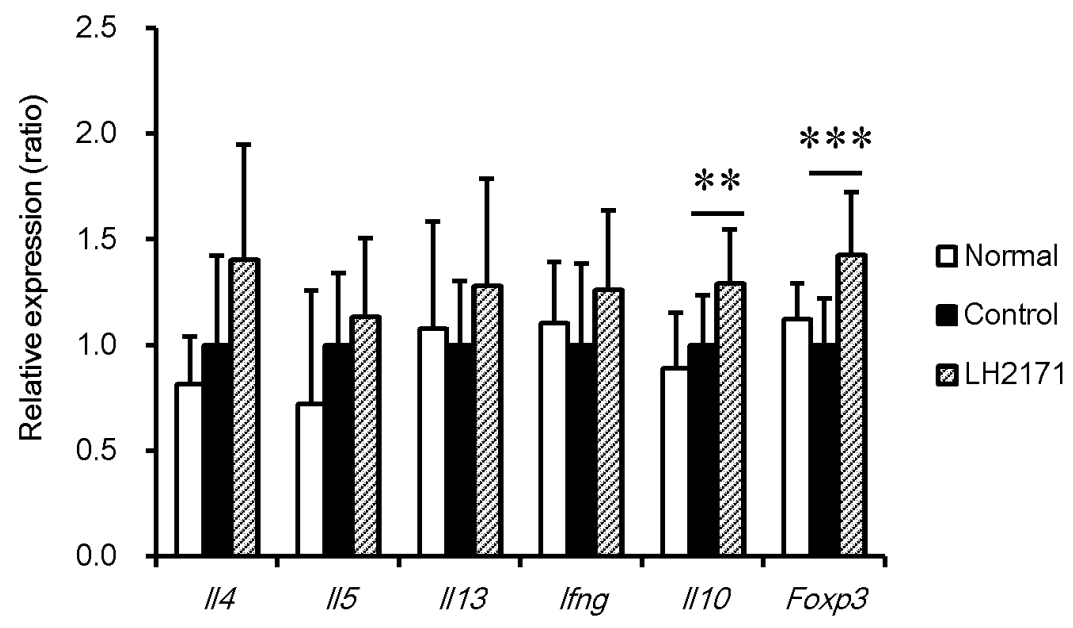

Figure 5. Effect of LH2171 on allergy-related cytokine and transcription factor expression in the Peyer's patch. The Peyer's patch was collected from mice 21 days after the first immunization. The expression levels of cytokine and transcription factor genes were determined by qRT-PCR. Data are shown as the means $\pm \mathrm{SD}$ (normal group, $\mathrm{n}=3$; control group, $\mathrm{n}=12$; LH2171 group, $\mathrm{n}=12$ ). The LH2171 group was compared with the control group by Student's $t$-test $(* * P<0.01, * * * P<0.001)$.

\section{DISCUSSION}

In the present study, we revealed that LH2171 is effective in a murine allergy model. There are many reports that LAB, a component of some foods, alleviate allergy in a murine pollen allergeninduced allergy model. Nonetheless, most reports used ovalbumin (the main protein found in egg whites and widely used in the field of immunological research) as an antigen, instead of pollen [22-28]. On the other hand, our present study demonstrated that LH2171 is effective in a murine model of allergy induced by pollen antigen. Indeed, a few reports showed that LAB were effective in an allergy model using pollen [29, 30], but the effectiveness of live LAB was examined. In this study, LH2171 was hypothesized to exert its effect in a non-living form in the murine intestine, because LH2171 is highly intolerant of intestinal digestive juices. In fact, our previous study showed that heat-killed LH2171 significantly suppressed the proliferation and inflammatory cytokine production of immune cells in vitro [16], although the active components of LH2171 contributing to the immune suppressive activity have not been clarified. Based on these findings, a wide range of products containing LH2171 as an effective biogenic for pollen allergy are expected to be developed in the future.

Allergic reactions such as sneezing, runny nose, and congestion of eyes are generally caused by a sequence of pathways: the production of cytokines by Th 2 cells, activation of B cells by Th2 cytokines and Th2 cells themselves, antibody production from B cells, degranulation of mast cells by binding of antigen and antigen specific-antibody to their surface, and allergic reactions caused by inflammatory substances such as histamine contained in degranulation [31-34]. Th2 cytokines, 
including IL-4, IL-5, and IL-13, play important roles in establishing allergies and are required during the production of antigen-specific IgG or IgE [35], while Th1 cytokines including IFN- $\gamma$ are relatively suppressed and vice versa. Anti-inflammatory cytokine IL-10, mainly produced by regulatory $\mathrm{T}$ cells (Tregs), also suppresses Th2 cytokine production and the subsequent antigenspecific antibody production and allergy symptoms [36-38]. Therefore, the alleviation of allergy likely occurs within these pathways. In the present study, oral administration of LH2171 decreased antigen-specific IgG1 and total IgE levels in the blood. Moreover, LH2171 suppressed antigeninduced Th2 cytokine production and proliferation of LN cells. Proliferation of immune cells is a primary indicator of inflammatory immune response, because proliferation generally occurs immediately following cellular encounter with antigen and immune system activation [16, 39]. These results suggested that LH2171 firstly works on Th2 cells to suppress cytokine production, thereby inhibiting antibody production and lowering the antibody concentration in the blood. Th2 cells are located upstream of B cells and mast cells in the above-described series of allergic reaction pathways; thus, LH2171 acting on Th2 cells may fundamentally resolve allergies.

In order to investigate the mechanism of suppression of antibody and Th2 cytokine production by LH2171, we analyzed PP, where immune cells frequently take up antigens and where the orally administered LH2171 was likely to first make contact with immune cells. The results in Figure 4 prompted us to consider whether day 42 was an appropriate time point for evaluating the immunosuppressive ability of LH2171: levels of anti-inflammatory cytokine IL-10 were lower in the LH2171 group than in the control group, probably because inflammatory reaction, such as Th2 cytokine production and proliferation of immune cells, was already suppressed by LH2171, and anti-inflammatory factors such as IL-10 would not need to be produced at day 42. In fact, our previous study and some other reports showed that even though LAB have the ability to induce anti-inflammatory factors such as IL-10 and Foxp3 in normal conditions or prior to inflammation, they do not always increase these factors in the inflammatory phase or affected organ [40-42]. Therefore, we evaluated the expression of allergy-related cytokines and transcription factors in PP at day 21 after the first immunization, an earlier time point. As expected, our analysis revealed that LH2171 increased the expression of IL-10 and Foxp3, a marker of Tregs, which regulate the function of other immune cells including Th2 and Th1 and produce IL-10 [36, 38]. These results suggest that this increase in Tregs and IL-10 production might be correlated with suppression of Th2 activation by LH2171. Tregs have been reported to be effective for various diseases and syndromes, such as autoimmune diseases-including multiple sclerosis, systemic lupus erythematosus, rheumatoid arthritis, inflammatory bowel disease, and psoriasis [43] — obesitylinked insulin resistance and diabetic nephropathy [44], and anxiety and depression-like behaviors [45]. In addition, LH2171 is already used for food production as a starter bacterium for Gouda cheese. Considering these findings, LH2171 might greatly contribute to the prevention of allergy and various diseases. Nonetheless, the pathways through which LH2171 increases Tregs and IL10 production, acts directly on $\mathrm{T}$ cells, and is incorporated into antigen-presenting cells remain to be investigated. 


\section{CONCLUSION}

In conclusion, our study demonstrated that the oral administration of LH2171 significantly decreases antigen-specific antibody and total $\mathrm{IgE}$ in plasma and reduces the production of $\mathrm{Th} 2$ cytokines by submandibular LN cells in a murine model of pollen allergy. Furthermore, LH2171 increased the expression of anti-inflammatory factors such as IL-10 and Foxp3 in PP, which correlates with the preventive effects of LH2171 against allergic responses. These findings suggest that LH2171 could exert a preventive or mitigating effect for individuals suffering from allergies.

List of abbreviations: AIN, American Institute of Nutrition; ELISA, enzyme-linked immunosorbent assay; LAB, lactic acid bacteria; LH2171, Lactobacillus helveticus SBT2171; LNs, lymph nodes; LPS, lipopolysaccharide; PBS, phosphate-buffered saline; PCR, polymerase chain reaction; PP, Peyer's patch; RT, room temperature; SP, spleen; Tregs, regulatory T cells; TweenPBS, 0.05\% Tween 20 in PBS.

Author's contributions: M. Yamashita designed the study and wrote the initial draft of the manuscript. T. Kabuki also designed the study and contributed to interpretation of data. K. Matsumoto, N. Matsumoto, and E. Kobatake contributed to analysis. All other authors contributed to data collection and interpretation and critically reviewed the manuscript. All authors approved the final version of the manuscript and agree to be accountable for all aspects of the work in ensuring that questions related to the accuracy or integrity of any part of the work are appropriately investigated and resolved.

Competing interests: M. Y., K. M., N. M., E. K., and T. K. are employees of Megmilk Snow Brand Co., Ltd. This study was funded by Megmilk Snow Brand Co., Ltd.

Acknowledgments: We would like to thank Editage (www.editage.jp) for English language editing.

\section{REFERENCES}

1. D’Amato G, Holgate ST, Pawankar R, Ledford DK, Cecchi L, Al-Ahmad M, Al-Enezi F, et al.: Meteorological conditions, climate change, new emerging factors, and asthma and related allergic disorders. A statement of the World Allergy Organization. World Allergy Organ J 2015, 8(1): 1-52.

2. Okubo K, Kurono Y, Ichimura K, Enomoto T, Okamoto Y, Kawauchi H, Suzaki H, et al.: Japanese guidelines for allergic rhinitis 2017. Allergol Int 2017, 66(2): 205-219.

3. Yamada T, Saito H, Fujieda S: Present state of Japanese cedar pollinosis: The national affliction. J Allergy Clin Immunol 2014, 133(3): 632-639.

4. Murooka Y, Yamshita M: Traditional healthful fermented products of Japan. J Ind Microbiol Biotechnol 2008, 35(8): 791-798.

5. Parvez S, Malik KA, Ah Kang S, Kim HY: Probiotics and their fermented food products are beneficial for health. J Appl Microbiol 2006, 100(6): 1171-1185

6. Mitsuoka T: Development of functional foods. Biosci Microbiota Food Health 2014, 
33(3): 117-128.

7. Akatsu H, Iwabuchi N, Xiao J, Matsuyama Z, Kurihara R, Okuda K, Yamamoto T, et al.: Clinical effects of probiotic Bifidobacterium longum BB536 on immune function and intestinal microbiota in elderly patients receiving enteral tube feeding. JPEN J Parenter Enteral Nutr 2013, 37(5): 631-640.

8. Namba K, Hatano M, Yaeshima T, Takase M, Suzuki K: Effects of Bifidobacterium longum BB536 administration on influenza infection, influenza vaccine antibody titer, and cell-mediated immunity in the elderly. Biosci Biotechnol Biochem 2010, 74(5): 939945.

9. Wang Y, Liu L, Moore DJ, Shen X, Peek RM, Acra SA, Li H, et al.: An LGG-derived protein promotes IgA production through upregulation of APRIL expression in intestinal epithelial cells. Mucosal Immunol 2017, 10(2): 373-384.

10. Shandilya UK, Sharma A, Kapila R, Kansal VK: Probiotic Dahi containing Lactobacillus acidophilus and Bifidobacterium bifidum modulates immunoglobulin levels and cytokines expression in whey proteins sensitised mice. J Sci Food Agric 2016, 96(9): 3180-3187.

11. Yamamoto K, Yokoyama K, Matsukawa T, Kato S, Kato S, Yamada K, Hirota T: Efficacy of prolonged ingestion of Lactobacillus acidophilus L-92 in adult patients with atopic dermatitis. J Dairy Sci 2016, 99(7): 5039-5046.

12. Amdekar S, Singh V, Singh R, Sharma P, Keshav P, Kumar A: Lactobacillus casei reduces the inflammatory joint damage associated with collagen-induced arthritis (CIA) by reducing the pro-inflammatory cytokines - Lactobacillus casei: COX-2 inhibitor. J Clin Immunol 2011, 31(2): 147-154.

13. Kato I, Endo-Tanaka K, Yokokura T: Suppressive effects of the oral administration of Lactobacillus casei on type II collagen-induced arthritis in DBA/1 mice. Life Sci 1998, 3(8): 635-644.

14. So JS, Lee CG, Kwon HK, Yi HJ, Chae CS, Park JA, Hwang KC, et al.: Lactobacillus casei potentiates induction of oral tolerance in experimental arthritis. Mol Immunol 2008, 46(1): 172-180.

15. Sasaki M, Bosman BW, Tan PST: A new, broad-substrate-specificity aminopeptidase from the dairy organism Lactobacillus helveticus SBT2171. Microbiology 1996, 142(4): 799-808.

16. Yamashita M, Ukibe K, Uenishi H, Hosoya T, Sakai F, Kadooka Y: Lactobacillus helveticus SBT2171, a cheese starter, regulates proliferation and cytokine production of immune cells. J Dairy Sci 2014, 97(8): 4772-4779.

17. Hosoya T, Sakai F, Yamashita M, Shiozaki T, Endo T, Ukibe K, Uenishi H, et al.: Lactobacillus helveticus SBT2171 inhibits lymphocyte proliferation by regulation of the JNK signaling pathway. PLoS ONE 2014, 9(9): 1-9.

18. Yamashita M, Matsumoto K, Endo T, Ukibe K, Hosoya T, Matsubara Y, Nakagawa H, et al.: Preventive effect of Lactobacillus helveticus SBT2171 on collagen-induced arthritis in mice. Front Microbiol 2017, 8: 1159.

19. Yamashita M, Ukibe K, Matsubara Y, Hosoya T, Sakai F, Kon S, Arima Y, et al.: 
Lactobacillus helveticus SBT2171 attenuates experimental autoimmune encephalomyelitis in mice. Front Microbiol 2018, 8: 2596.

20. Reeves PG: Components of the AIN-93 diets as improvements in the AIN-76A. J Nutr 1997, 127(5): 838-841.

21. Fujimura T, Fujinami K, Ishikawa R, Tateno M, Tahara Y, Okumura Y, Ohta H, et al.: Recombinant fusion allergens, Cry j 1 and Cry $\mathrm{j} 2$ from Japanese cedar pollen, conjugated with polyethylene glycol potentiate the attenuation of Cry $\mathrm{j} 1$-specific IgE production in Cry j 1-sensitized mice and Japanese cedar pollen allergen-sensitized monkeys. Int Arch Allergy Immunol 2015, 168(1): 32-43.

22. Inoue $\mathrm{Y}$, Iwabuchi N, Xiao JZ, Yaeshima T, Iwatsuki K: Suppressive effects of Bifidobacterium breve strain M-16V on T-helper type 2 immune responses in a murine model. Biol Pharm Bull 2009, 32(4): 760-763.

23. Kang H, Myung EJ, Ahn KS, Eom HJ, Han NS, Kim YB, Kim YJ, et al.: Induction of Th1 cytokines by Leuconostoc mesenteroides subsp. mesenteroides (KCTC 3100) under Th2-type conditions and the requirement of NF- $\mathrm{BB}$ and p38/JNK. Cytokine 2009, 46(2): 283-289.

24. Nonaka Y, Izumo T, Izumi F, Maekawa T, Shibata H, Nakano A, Kishi A, et al.: Antiallergic effects of Lactobacillus pentosus strain S-PT84 mediated by modulation of Th1/Th2 immunobalance and induction of IL-10 production. Int Arch Allergy Immunol 2008, 145(3): 249-257.

25. Ohno H, Tsunemine S, Isa Y, Shimakawa M, Yamamura H: Oral administration of Bifidobacterium bifidum G9-1 suppresses total and antigen specific immunoglobulin E production in mice. Biol Pharm Bull 2005, 28(8): 1462-1466.

26. Segawa S, Nakakita Y, Takata Y, Wakita Y, Kaneko T, Kaneda H, Watari J, et al.: Effect of oral administration of heat-killed Lactobacillus brevis SBC8803 on total and ovalbumin-specific immunoglobulin E production through the improvement of Th1/Th2 balance. Int J Food Microbiol 2008, 121(1): 1-10.

27. Shida K, Takahashi R, Iwadate E, Takamizawa K, Yasui H, Sato T, Habu S, et al.: Lactobacillus casei strain Shirota suppresses serum immunoglobulin E and immunoglobulin G1 responses and systemic anaphylaxis in a food allergy model. Clin Exp Allergy 2002, 32(4): 563-570.

28. Sunada Y, Nakamura S, Kamei C: Effects of Lactobacillus acidophilus strain L-55 on experimental allergic rhinitis in BALB/c mice. Biol Pharm Bull 2007, 30(11): 21632166.

29. Repa A, Grangette C, Daniel C, Hochreiter R, Hoffmann-Sommergruber K, Thalhamer $\mathrm{J}$, Kraft D, et al.: Mucosal co-application of lactic acid bacteria and allergen induces counter-regulatory immune responses in a murine model of birch pollen allergy. Vaccine 2003, 22(1): 87-95.

30. Schabussova I, Hufnagl K, Tang MLK, Hoflehner E, Wagner A, Loupal G, Nutten S, et al.: Perinatal maternal administration of Lactobacillus paracasei NCC 2461 prevents allergic inflammation in a mouse model of birch pollen allergy. PLoS ONE 2012, 7(7): e40271. 
31. Kay AB: Allergy and allergic diseases. First of two parts. N Engl J Med 2001, 344(1): 30-37.

32. Kay AB: Allergy and allergic disease. Second of two parts. N Engl J Med 2001, 344(2): 109-113.

33. Lee NP, Arriola ER: How to treat allergic rhinitis. West J Med 1999, 171(1): 31-34.

34. Romagnani S: The role of lymphocytes in allergic disease. J Allergy Clin Immunol 2000, 105(3): 399-408.

35. Lehman JM, Blaiss MS: Selecting the optimal oral antihistamine for patients with allergic rhinitis. Drugs 2006, 66(18): 2309-2319.

36. Akbari O, Stock P, DeKruyff RH, Umetsu DT: Role of regulatory T cells in allergy and asthma. Curr Opin Immunol 2003, 15(6): 627-633.

37. Ling EM, Smith T, Nguyen XD, Pridgeon C, Dallman M, Arbery J, Carr VA, et al.: Relation of $\mathrm{CD}^{+} \mathrm{CD} 25^{+}$regulatory T-cell suppression of allergen-driven T-cell activation to atopic status and expression of allergic disease. Lancet 2004, 363(9409): 608-615.

38. Teh PP, Vasanthakumar A, Kallies A: Development and function of effector regulatory $\mathrm{T}$ cells. Prog Mol Biol Transl Sci 2015, 136: 155-174.

39. Popple A, Williams J, Maxwell G, Gellatly N, Dearman RJ, Kimber I: The lymphocyte transformation test in allergic contact dermatitis: New opportunities. J Immunotoxicol 2016, 13(1): 84-91.

40. Hosoya T, Ogawa A, Sakai F, Kadooka Y: A cheese-containing diet modulates immune responses and alleviates dextran sodium sulfate-induced colitis in mice. J Dairy Sci 2012, 95(6): 2810-2818.

41. Takata K, Kinoshita M, Okuno T, Moriya M, Kohda T, Honorat JA, Sugimoto T, et al.: The lactic acid bacterium Pediococcus acidilactici suppresses autoimmune encephalomyelitis by inducing IL-10-producing regulatory T cells. PLoS One 2011, 6(11): e27644.

42. Wasilewska E, Zlotkowska D, Wroblewska B: Yogurt starter cultures of Streptococcus thermophilus and Lactobacillus bulgaricus ameliorate symptoms and modulate the immune response in a mouse model of dextran sulfate sodium-induced colitis. J Dairy Sci 2019, 102(1): 37-53.

43. Soltanzadeh-Yamchi M, Shahbazi M, Aslani S, Mohammadnia-Afrouzi M: MicroRNA signature of regulatory T cells in health and autoimmunity. Biomed Pharmacother 2018, 100: 316-323.

44. Eller K, Kirsch A, Wolf AM, Sopper S, Tagwerker A, Stanzl U, Wolf D, et al.: Potential role of regulatory $\mathrm{T}$ cells in reversing obesity-linked insulin resistance and diabetic nephropathy. Diabetes 2011, 60(11): 2954-2962.

45. Kim SJ, Lee H, Lee G, Oh SJ, Shin MK, Shim I, Bae H: $\mathrm{CD} 4{ }^{+} \mathrm{CD} 25^{+}$regulatory T cell depletion modulates anxiety and depression-like behaviors in mice. PLoS ONE 2012, 7(7): e42054. 